\title{
Isavuconazole in the treatment of invasive aspergillosis and mucormycosis infections
}

This article was published in the following Dove Press journal:

Infection and Drug Resistance

2 June 2016

Number of times this article has been viewed

\author{
Monica A Donnelley ${ }^{1,2}$ \\ Elizabeth S Zhu' \\ George R Thompson 3rd ${ }^{3}$ \\ 'Department of Inpatient Pharmacy, \\ University of California - Davis, \\ Sacramento, ${ }^{2}$ Department of Clinical \\ Sciences, Touro University College \\ of Pharmacy, Vallejo, ${ }^{3}$ Department \\ of Medicine, Division of Infectious \\ Diseases, University of California - \\ Davis, Davis, CA, USA
}

Correspondence: George R Thompson 3rd Department of Medicine, Division of Infectious Diseases, University of California - Davis, One Shields Ave, Tupper Hall, Rm 3138, Davis, CA 956I6, USA

Tel +I 5307523545

Fax + I 5307528692

Email grthompson@ucdavis.edu
Abstract: We have a limited arsenal with which to treat invasive fungal infections caused by Aspergillus and Mucorales. The morbidity and mortality for both pathogens remains high. A triazole antifungal, isavuconazole, was recently granted approval by the US Food and Drug Administration and the European Medicines Agency for the treatment of invasive aspergillosis and mucormycosis. A randomized double-blind comparison trial for the treatment of invasive aspergillosis found isavuconazole noninferior to voriconazole. A separate, open-label study evaluating the efficacy of isavuconazole in the treatment of mucormycosis found comparable response rates to amphotericin $\mathrm{B}$ and posaconazole treated historical controls. The prodrug isavuconazonium sulfate is commercially available in both an oral and intravenous formulation and is generally well tolerated. Isavuconazole's broad spectrum of activity, limited side effect profile, and favorable pharmacokinetics will likely solidify its place in therapy.

Keywords: isavuconazonium, antifungal, zygomycosis, mucor, mold infection, pharmacokinetics, pharmacodynamics, clinical efficacy, therapy, new agent

\section{Introduction}

Invasive fungal infections are responsible for significant morbidity and mortality, particularly in immunocompromised hosts. Although mortality from invasive candidiasis has decreased in recent years, an overall increase in the number of deaths from invasive fungal infections has been noted, largely due to invasive aspergillosis and other molds. ${ }^{1-4}$ The number of patients at risk has also increased as a greater number of patients are exposed to immunosuppressive therapy and more intensive chemotherapy regimens.

Currently available antifungal agents are useful for the management of the majority of the mycoses encountered in routine practice, however, significant differences exist between the triazoles in regard to efficacy, pharmacokinetics and pharmacodynamics, drug-drug interactions, the need for therapeutic drug monitoring (TDM), and toxicity. ${ }^{5}$ The use of amphotericin B is problematic given the incidence of nephrotoxicity and requirement for intravenous (IV) administration, and flucytosine is not useful as monotherapy for most infections and lacks meaningful activity against a multitude of others (including Aspergillus spp. and the Mucorales). The echinocandins are excellent agents in the fight against invasive candidal infections, ${ }^{6}$ however, their use in the treatment of aspergillosis is limited to combination or salvage therapy, and they are used solely in combination with polyenes in the treatment of mucormycosis.

The water-soluble prodrug isavuconazonium sulfate was developed specifically to facilitate IV administration without the need for potentially nephrotoxic excipients such 
as $\beta$-cyclodextrin, and displays excellent oral bioavailability $(\sim 98 \%) .^{7}$ This review will summarize the role of this new triazole in the treatment of Aspergillus and Mucorales infections.

\section{In vitro activity and animal models}

The triazoles, including isavuconazole, exert their effects within the fungal cell membrane. The inhibition of cytochrome P450 (CYP)-dependent 14- $\alpha$-demethylase by azoles prevents the conversion of lanosterol to ergosterol. This mechanism results in the accumulation of toxic methyl sterols and resultant inhibition of fungal cell growth and replication. The side arm of the active isavuconazole molecule allows for greater affinity of isavuconazole for the binding pocket in the fungal CYP51 protein increasing the antifungal spectrum and conferring activity against some isolates resistant to other triazoles. ${ }^{8}$

The in vitro activity of isavuconazole has been demonstrated against molds, yeasts, and dimorphic fungi using standardized methodologies as suggested by the Clinical and Laboratory Standards Institute. ${ }^{9-16}$ Activity is seen against most Aspergillus spp. including A. fumigatus, A. flavus, A. terreus, and $A$. niger ${ }^{16}$ with minimum inhibitory concentration to inhibit $50 \%$ of the organism growth $\left(\mathrm{MIC}_{50} \mathrm{~s}\right)$ of $0.25-1 \mu \mathrm{g} / \mathrm{mL}$. In recent years, the emergence of azole resistant Aspergillus spp. has become a threat to patients with the majority of clinical cases to date reported from western Europe. ${ }^{17}$ In these resistant isolates, mutations within the CYP51A gene (which encodes the enzyme targeted by azoles) or within the gene promoter, have been identified that reduce drug affinity or increase target quantity conferring azole resistance. Aspergillus isolates with elevated minimal inhibitory concentrations (MICs) to posaconazole and voriconazole have been evaluated for isavuconazole susceptibility and crossresistance between azoles has been found inconsistent. ${ }^{18,19}$ For example, Aspergillus isolates with the alterations L98, G138, Y431C, or G434C show elevated MICs to all triazoles including isavuconazole, however, the MICs of posaconazole are much lower than those of voriconazole and isavuconazole. In contrast, isolates with the G54 mutation show lower voriconazole and isavuconazole MICs compared to posaconazole, and MICs are variable among azoles in isolates with the M220 alteration. ${ }^{18}$ These results suggest a potential role for routine susceptibility testing now that multiple azoles are available for clinical use. Further work will need to be performed to guide the clinician and to ensure these in vitro findings are indicative of in vivo efficacy.

Genera in the family Mucoraceae (order Mucorales) include Rhizopus, Cunninghamella, Lictheimia (formerly
Absidia), Mucor, and Rhizomucor. Prior to the availability of isavuconazole, these organisms were resistant to all azoles with the exception of posaconazole. ${ }^{9}$ The in vitro activity of isavuconazole against the Mucorales is more variable with $\mathrm{MIC}_{50}$ values ranging from 1 to $4 \mu \mathrm{g} / \mathrm{mL}$ depending on the specific species and isolate tested..$^{20}$ For example, Rhizopus isolates have MIC values to isavuconazole as low as $0.12 \mu \mathrm{g} / \mathrm{mL}$ with others as high as $32 \mu \mathrm{g} / \mathrm{mL}$; findings suggesting susceptibility testing should be performed on all Mucorales isolates in attempts to maximize pharmacokinetic/pharmacodynamic parameters during treatment.

Isavuconazole has fungistatic activity against yeasts such as Candida and Cryptococcus; however, appears to be fungicidal against Aspergillus spp. The area under the curve to $\mathrm{MIC}$ ratio $(\mathrm{AUC} / \mathrm{MIC})$ is the primary predictor of drug efficacy in animal models of aspergillosis. ${ }^{21-23}$ Animal models have thus far consisted primarily of dose-finding experiments given the differences in metabolism between murine models and humans, and assessments of efficacy using a number of different fungal species and strains. This work has thus far focused on Candida, Cryptococcus, Aspergillus, and the Mucorales, with other invasive molds, yeasts, and endemic mycoses to be examined in the near future..$^{21,23-29}$

\section{Pharmacology}

Isavuconazonium is a prodrug of isavuconazole and is a water-soluble molecule consisting of a N-(3acetoxypropyl)-N-methylamino-carboxymethyl group attached to isavuconazole via an ester moiety (Figure 1). ${ }^{30}$ Isavuconazonium sulfate is the commercially available prodrug formulation and is available in both an oral capsule and IV lyophilized powder., ${ }^{9,31}$ The IV formulation of isavuconazonium sulfate is $372 \mathrm{mg}$, which is equivalent to $200 \mathrm{mg}$ of isavuconazole. The oral capsule has $186 \mathrm{mg}$ of isavuconazonium sulfate leading to $100 \mathrm{mg}$ of isavuconazole. ${ }^{31}$ Special attention should be paid to initiation of this agent as dosing isavuconazonium sulfate involves a loading dose followed by once daily administration. The loading dose is $372 \mathrm{mg}$ of isavuconazonium sulfate $(200 \mathrm{mg}$ of isavuconazole) every 8 hours for 2 days, then $372 \mathrm{mg}$ of isavuconazonium sulfate ( $200 \mathrm{mg}$ of isavuconazole) once daily. When transitioning from an IV to an oral formulation, a repeat loading dose is not necessary. ${ }^{32}$ Dosing in the pediatric population has not been determined (trial is ongoing) and no recommendations can be given for this population.

Isavuconazonium's oral formulation is well absorbed and has excellent bioavailability (98\%). In preclinical studies 
isavuconazole exhibited linear and dose-proportional pharmacokinetics with low interpatient variability $(2.5 \pm 1.0 \mu \mathrm{g} / \mathrm{mL}) .{ }^{33}$ Food has no effect on the pharmacokinetics of isavuconazonium and is not a limitation during its administration. ${ }^{31}$ The volume of distribution for isavuconazonium is large at $450 \mathrm{~L}$ and the drug half-life is 130 hours. Thus tissue levels persist long after plasma levels are undetectable. ${ }^{29}$

The IV prodrug formulation is water soluble, unlike voriconazole or posaconazole, and does not require cyclodextrin for solubilization. Following IV infusion, the prodrug and inactive cleavage product are undetectable after 30 minutes. With oral administration, the maximum concentration $\left(\mathrm{C}_{\max }\right)$ is reached within 2-3 hours; the prodrug and cleavage products are undetectable with oral administration. Elimination occurs primarily via the fecal route while renal excretion is $<1 \%$ of the dose administered suggesting little utility in the treatment of fungal urinary tract infections. ${ }^{32,34}$

The metabolism of isavuconazonium is through rapid hydrolyzation, mainly by serum butylcholinesterase, to isavuconazole; CYP3A4, CYP3A5, and uridine diphosphateglucuronosyl transferase are substrates of isavuconazole. ${ }^{32}$ The metabolites do not possess antifungal activity. In those with mild-to-moderate hepatic impairment (Child-Pugh class A or B) dosage adjustment is not needed. No data are

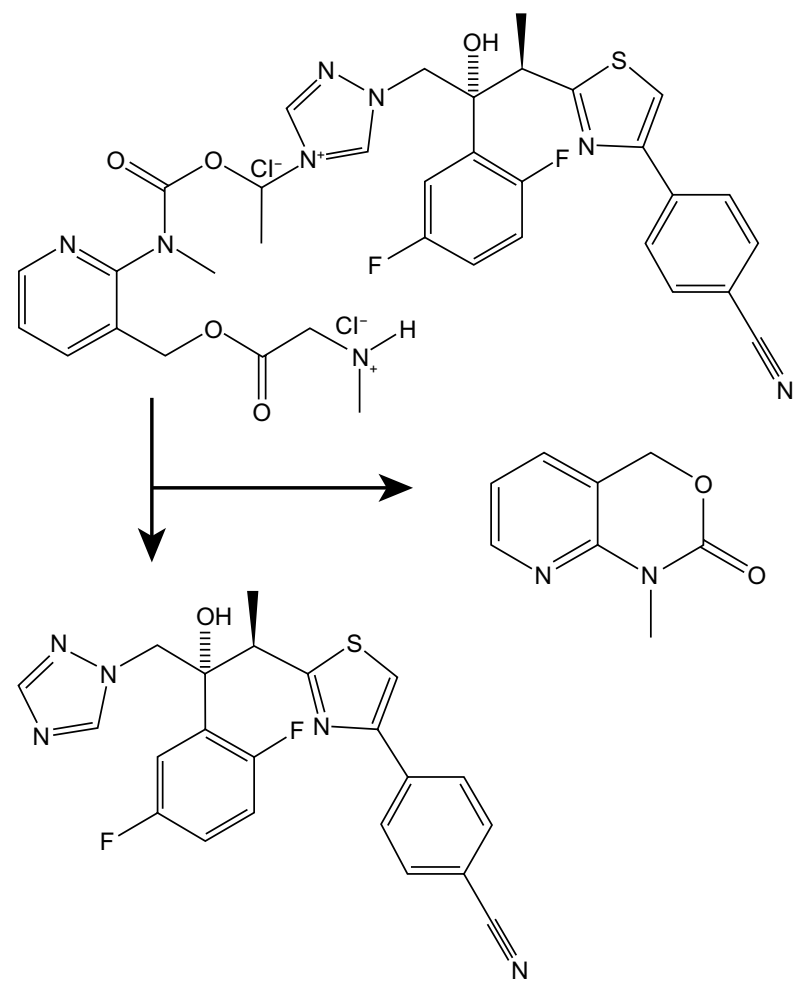

Figure I Metabolism of isavuconazonium by plasma esterases into an inactive byproduct and the active antifungal isavuconazole. available for dosing in those with Child-Pugh class C liver disease.

Renal impairment does not result in changes in the $\mathrm{C}_{\max }$ or AUC of isavuconazole and dosage adjustment is not needed in this group either. Pharmacokinetic studies have not been performed in dialysis patients to date, however, it is likely not cleared by hemodialysis or continuous hemofiltration. ${ }^{8}$

Isavuconazole is a pregnancy class $\mathrm{C}$ drug and should not be administered to pregnant women. Additionally, it is present in breast milk and should not be given to women who are breastfeeding.

\section{Drug-drug interactions}

The triazole antifungal agents demonstrate significant drug-drug interactions that may adversely affect patient outcomes. ${ }^{35}$ Each patient's current medications should be reviewed for potentially deleterious drug interactions. As a class, these include altered serum levels of the azoles and of coadministered agents including calcineurin inhibitors and mammalian target of rapamycin inhibitor immunosuppressive agents, psychiatric and neurotropic medications, some benzodiazepines, digoxin, and antiretroviral agents among others. ${ }^{36-52}$ All of the azoles have important interactions via the CYP450 enzymes, notably CYP3A4.

Inhibitors of CYP3A4 lead to increased levels of isavuconazole and should be used with caution (Table 1). Inducers of CYP3A4 include rifampin, carbamazepine, barbiturates among others and significantly reduce serum levels of isavuconazole and concurrent administration should be avoided.

Isavuconazole is a moderate inhibitor of CYP3A4 and impairs the metabolism of sirolimus, tacrolimus, and cyclosporine causing higher levels of these immunosuppressive medications and warranting drug monitoring. ${ }^{53}$

TDM is routinely used for voriconazole, and in selected cases is used for fluconazole, itraconazole, and posaconazole. ${ }^{54}$

Table I Effect of isavuconazole compared to voriconazole on CYP substrates

\begin{tabular}{llll}
\hline CYP & Substrate & Isavuconazole & Voriconazole \\
\hline 3A4 & Midazolam & $\uparrow 2.05$-fold & $\uparrow 10.3$-fold \\
& Sirolimus & $\uparrow I .84$-fold & $\uparrow I$ II.0-fold \\
IA2 & Caffeine & NCS & NCS \\
2 C8 & Repaglinide & NCS & NCS \\
2C9 & Warfarin & NCS & $\uparrow 2$-fold (PT) \\
2CI9 & Omeprazole & NCS & $\uparrow 4$-fold \\
2B6 & Buproprion & $\downarrow 42 \%$ & $\uparrow I .3$-fold \\
2D6 & Dextromethorphan & NCS & NCS \\
\hline
\end{tabular}

Abbreviations: CYP, cytochrome; NCS, not clinically significant; PT, prothrombin time. 
No recommendation can be made for or against routine isavuconazole TDM, however, in some clinical cases it is clear TDM will be needed (eg, bidirectional drug-drug interactions, during care of the morbidly obese).

\section{Current treatments for invasive aspergillosis and mucormycosis}

Both invasive aspergillosis and mucormycosis are devastating infections and improving patient outcomes requires aggressive screening of at-risk populations to ensure early diagnosis and the rapid initiation of appropriate antifungal therapy. The currently recommended first-line therapy in the treatment of invasive aspergillosis is voriconazole,${ }^{55}$ with amphotericin B formulations and echinocandins reserved for those intolerant or refractory to this agent. The Mucorales are treated by amphotericin B with adjunctive surgical intervention when possible, yet mortality remains between $50 \%$ and $100 \%{ }^{56}$ Due to the aforementioned problems with currently available antifungal agents, isavuconazole was developed specifically to provide advantages over currently available antifungal compounds and has now been studied in three Phase III trials: 1) the SECURE trial - isavuconazole versus voriconazole for primary treatment of invasive mold disease caused by Aspergillus and other filamentous fungi; 2) the VITAL trial - an open label study of isavuconazole in the treatment of patients with aspergillosis and renal impairment or of patients with invasive fungal disease caused by rare molds, yeasts, or dimorphic fungi; and 3) Isavuconazole (BAL8557) in the treatment of candidemia and other invasive Candida infections. Together these trials provide important information on the efficacy, safety, and clinical use of isavuconazole.

\section{Clinical efficacy}

The SECURE study was a Phase III, double-blind, randomized noninferiority study to compare the efficacy and safety of isavuconazole to voriconazole in the primary treatment of Aspergillus spp. or other filamentous fungi. ${ }^{57}$ The primary endpoint was all-cause mortality at day 42 in the intentionto-treat (ITT) population. The main secondary endpoint was a composite of clinical, mycological, radiological, and overall response, as assessed by a blinded data-review committee (DRC). Patients randomized to isavuconazole were administered $200 \mathrm{mg}$ IV three times a day for days 1 and 2 , followed by either IV or oral $200 \mathrm{mg}$ once daily. Those patients receiving voriconazole were administered $6 \mathrm{mg} /$ $\mathrm{kg}$ IV twice daily on day 1 , followed by $4 \mathrm{mg} / \mathrm{kg}$ IV twice daily on day 2 , then either $4 \mathrm{mg} / \mathrm{kg}$ IV twice daily or 200 mg oral twice daily from day 3 onwards. Overall 516 adult patients were randomized and treated (258 per group) in the
ITT population. The baseline demographics were similar between the two treatment groups, although there were fewer males in the isavuconazole group and more acute myeloid leukemia patients in the voriconazole group. All-cause mortality through day 42 for the ITT population was $18.6 \%$ (isavuconazole) and 20.2\% (voriconazole; adjusted treatment difference: $-1.0 \%$; 95\% confidence interval: $-7.8 \%, 5.7 \%$ ). Overall response rates at end of treatment in the modified ITT population were $35.0 \%$ (isavuconazole) and $36.4 \%$ (voriconazole). The study concluded that isavuconazole was noninferior to voriconazole for the primary treatment of invasive mold disease and the US Food and Drug Administration (FDA) and European Medicines Agency (EMA) have since licensed isavuconazole for the treatment of invasive aspergillosis.

The VITAL study was a Phase III, open-label, noncomparative trial evaluating the efficacy and safety of isavuconazole in patients with invasive aspergillosis with renal impairment or as primary or salvage treatment for mucormycosis. ${ }^{58}$ Isavuconazole $200 \mathrm{mg}$ (IV or oral) was given three times daily for six doses, followed by $200 \mathrm{mg} /$ day until invasive fungal disease resolution or $\geq 180$ days. Outcomes included mortality at day 42 and overall response as determined by an independent DRC. Of the 149 patients enrolled, 37 had proven $(86 \%)$ or probable $(14 \%)$ invasive mucormycosis by European Organization for Research and Treatment of Cancer/Mycoses Study Group criteria. ${ }^{59}$ Mortality at day 42 was $37.8 \%$. A matched case-control cohort of 33 patients treated with amphotericin B from the FungiScope registry was compared with 21 patients who received isavuconazole for the primary treatment of mucormycosis. Mortality rates were not significantly different between cases and controls (33.3\% and $41.3 \%$, respectively). A relationship between trough isavuconazole levels, fungal isolate MICs, and key outcomes could not be identified, possibly due to the relatively small number of patients with data available. Clinical responses occurred across the range of isavuconazole MICs and trough levels observed. The FDA has licensed isavuconazole for the treatment of mucormycosis, while the EMA has licensed isavuconazole in those for whom amphotericin B formulations are inappropriate. This difference suggests the continued opinion that amphotericin B formulations are first-line therapy for most cases of mucormycosis.

\section{Safety and tolerability}

Isavuconazole has been shown to be fairly well tolerated and safe thus far. In the SECURE study, patients on isavuconazole had significantly fewer study drug-related adverse events (AEs), as compared to voriconazole (42.4\% 
vs $59.8 \% ; P<0.001)$. AEs frequently associated with voriconazole such as visual disturbances, elevation in hepatic enzymes, and photosensitivity were significantly less common with isavuconazole. A few less frequent side effects noted were hypokalemia, peripheral edema, and infusion-related reactions. Acute respiratory distress, chills, dyspnea, and hypotension were among the infusion-related reactions.

Similar AE results were shown in the VITAL study. In total, 35/37 (95\%) patients experienced one or more AEs during treatment, and 28 (76\%) patients had serious AEs. The most common AEs were gastrointestinal complaints, such as nausea, vomiting, and diarrhea. Twenty-four patients discontinued isavuconazole treatment in the VITAL study, of which, six of these patients stopped due to AEs (relapse or progression of malignancy, two patients; acute liver injury, two patients; Escherichia coli bacteremia, one patient; and nausea, one patient). Overall, the AEs reported in the VITAL trial were similar in distribution to those reported in the SECURE invasive aspergillosis trial. There was no organ-specific pattern of serious AEs, however, as with all other azoles, liver enzymes should be monitored in patients receiving isavuconazole.

Isavuconazole differs uniquely from other triazoles in its class, in that it has dose- and concentration-dependent QTc interval shortening. ${ }^{7}$ QTc shortening up to $13 \mathrm{~ms}$ with 200 $\mathrm{mg}$ once daily dosing and $24.6 \mathrm{~ms}$ with $600 \mathrm{mg}$ daily have been shown in healthy volunteers. Inhibition of the L-type calcium channel is the proposed mechanism by which QTc shortening occurs. ${ }^{31}$ The clinical significance of this effect is unclear, however, those with familial short-QT syndrome should not receive isavuconazole.

\section{Patient-focused perspectives}

There are several advantages of isavuconazole, inherent in its pharmacology, which lend itself well to patient convenience and adherence. Unlike posaconazole and itraconazole, isavuconazole absorption is not affected by food and exhibits excellent oral bioavailability, close to $98 \% .^{7}$ These aspects lend themselves well for easy IV-to-PO conversion once a patient is clinically stable. Additionally, thus far there has been little interpatient variability suggesting routine TDM may not be necessary, however, further study in this area is needed before any recommendation for or against TDM can be made. The dosing of isavuconazole after the 48-hour loading period is once daily, with two capsules of $186 \mathrm{mg}$ isavuconazonium sulfate taken daily adding in patient convenience and potentially compliance. The IV formulation of isavuconazole does not require solubilization from a cyclodextrin vehicle, which eliminates concerns of nephrotoxicity that have arisen from the solubilizing agent including with IV voriconazole. ${ }^{60,61}$

The reduction in side effects in the isavuconazole treated patients compared to voriconazole treated patients in the SECURE study (difference of 17\%) does provide a significant advantage. Side effects of therapy may prolong hospital stays, delay additional chemotherapy while medication changes are made or patient symptoms resolve, and/or lead to patient distress (particularly visual hallucinations). The reduction in these untoward events, while maintaining antifungal efficacy, is a welcome additional to the antifungal armamentarium.

Long-term therapy with voriconazole has been linked with cutaneous malignancy, ${ }^{62-64}$ and hyperfluorosis with the development of painful exostoses in patients with impaired renal function. ${ }^{65,66}$ The side effects of isavuconazole with long-term use have not been fully evaluated, however, several patients in the VITAL study received prolonged therapy without side effects. Cutaneous malignancy has not been recognized to date, and hyperfluorosis with isavuconazole is unlikely given the differences in chemical structure between voriconazole and isavuconazole and that this phenomenon does not appear to be a class-effect. ${ }^{67}$

\section{Conclusion}

Isavuconazole has been recently approved by the FDA and EMA for the treatment of invasive aspergillosis and mucormycosis. This new triazole offers several advantages over existing antifungals including comparable efficacy to currently used agents with fewer side effects, advantageous pharmacokinetic parameters, and excellent bioavailability with oral administration. Future studies examining the role of isavuconazole in prophylaxis against invasive fungal infections, use in combination with other agents, and if TDM is necessary on a routine basis will further refine its role in the fight against these highly morbid and often lethal infections.

\section{Disclosure}

GRT has received research support from Wako Diagnostics, Merck, and Astellas, and served on the DRC and as a consultant for Astellas. The other authors report no conflicts of interest in this work.

\section{References}

1. Chamilos G, Luna M, Lewis RE, et al. Invasive fungal infections in patients with hematologic malignancies in a tertiary care cancer center: an autopsy study over a 15-year period (1989-2003). Haematologica. 2006;91:986-989. 
2. Pappas PG, Alexander BD, Andes DR, et al. Invasive fungal infections among organ transplant recipients: results of the Transplant-Associated Infection Surveillance Network (TRANSNET). Clin Infect Dis. 2010;50:1101-1111.

3. Kontoyiannis DP, Marr KA, Park BJ, et al. Prospective surveillance for invasive fungal infections in hematopoietic stem cell transplant recipients, 2001-2006: overview of the Transplant-Associated Infection Surveillance Network (TRANSNET) Database. Clin Infect Dis. 2010;50:1091-1100.

4. Pagano L, Caira M, Candoni A, et al. Invasive aspergillosis in patients with acute myeloid leukemia: a SEIFEM-2008 registry study. Haematologica. 2010;95:644-650.

5. Dodds-Ashley ES, Lewis RE, Lewis JSn, Martin C, Andes D. Pharmacology of systemic antifungal agents. Clin Infect Dis. 2006;43: S28-S39.

6. Andes DR, Safdar N, Baddley JW, et al. Impact of treatment strategy on outcomes in patients with candidemia and other forms of invasive candidiasis: a patient-level quantitative review of randomized trials. Clin Infect Dis. 2012;54:1110-1122.

7. Schmitt-Hoffmann A, Roos B, Heep M, et al. Single-ascending-dose pharmacokinetics and safety of the novel broad-spectrum antifungal triazole BAL4815 after intravenous infusions (50, 100, and 200 milligrams) and oral administrations (100, 200, and 400 milligrams) of its prodrug, BAL8557, in healthy volunteers. Antimicrob Agents Chemother. 2006;50:279-285.

8. Miceli MH, Kauffman CA. Isavuconazole: a new broad-spectrum triazole antifungal agent. Clin Infect Dis. 2015;61:1558-1565.

9. Thompson GR, 3rd, Wiederhold NP. Isavuconazole: a comprehensive review of spectrum of activity of a new triazole. Mycopathologia. 2010; 170:291-313

10. Pfaller MA, Rhomberg PR, Messer SA, Jones RN, Castanheira M. Isavuconazole, micafungin, and 8 comparator antifungal agents' susceptibility profiles for common and uncommon opportunistic fungi collected in 2013: temporal analysis of antifungal drug resistance using CLSI species-specific clinical breakpoints and proposed epidemiological cutoff values. Diagn Microbiol Infect Dis. 2015;82:303-313.

11. Espinel-Ingroff A, Chowdhary A, Gonzalez GM, et al. Multicenter study of isavuconazole MIC distributions and epidemiological cutoff values for the Cryptococcus neoformans-Cryptococcus gattii species complex using the CLSI M27-A3 broth microdilution method. Antimicrob Agents Chemother. 2015;59:666-668.

12. Thompson GR, 3rd, Wiederhold NP, Sutton DA, Fothergill A, Patterson TF. In vitro activity of isavuconazole against Trichosporon, Rhodotorula, Geotrichum, Saccharomyces and Pichia species. J Antimicrob Chemother. 2009;64:79-83.

13. Thompson GR 3rd, Wiederhold NP, Fothergill AW, Vallor AC, Wickes BL, Patterson TF. Antifungal susceptibilities among different serotypes of Cryptococcus gattii and Cryptococcus neoformans. Antimicrob Agents Chemother. 2009;53:309-311.

14. Thompson GR 3rd, Fothergill AW, Wiederhold NP, Vallor AC, Wickes BL, Patterson TF. Evaluation of Etest method for determining isavuconazole MICs against Cryptococcus gattii and Cryptococcus neoformans. Antimicrob Agents Chemother. 2008;52:2959-2961.

15. Datta K, Rhee P, Byrnes E 3rd, et al. Isavuconazole activity against Aspergillus lentulus, Neosartorya udagawae, and Cryptococcus gattii, emerging fungal pathogens with reduced azole susceptibility. $J$ Clin Microbiol. 2013;51:3090-3093.

16. Espinel-Ingroff A, Chowdhary A, Gonzalez GM, et al. Multicenter study of isavuconazole MIC distributions and epidemiological cutoff values for Aspergillus spp. for the CLSI M38-A2 broth microdilution method. Antimicrob Agents Chemother. 2013;57:3823-3828.

17. van der Linden JW, Arendrup MC, Warris A, et al. Prospective multicenter international surveillance of azole resistance in Aspergillus fumigatus. Emerg Infect Dis. 2015;21:1041-1044.

18. Gregson L, Goodwin J, Johnson A, et al. In vitro susceptibility of Aspergillus fumigatus to isavuconazole: correlation with itraconazole, voriconazole, and posaconazole. Antimicrob Agents Chemother. 2013;57:5778-5780.
19. Warn PA, Sharp A, Denning DW. In vitro activity of a new triazole BAL4815, the active component of BAL8557 (the water-soluble prodrug), against Aspergillus spp. J Antimicrob Chemother. 2006;57:135-138.

20. Arendrup MC, Jensen RH, Meletiadis J. In vitro activity of isavuconazole and comparators against clinical isolates of the Mucorales order. Antimicrob Agents Chemother. 2015;59:7735-7742.

21. Lepak AJ, Marchillo K, Vanhecker J, Andes DR. Isavuconazole (BAL4815) pharmacodynamic target determination in an in vivo murine model of invasive pulmonary aspergillosis against wild-type and cyp 51 mutant isolates of Aspergillus fumigatus. Antimicrob Agents Chemother. 2013;57:6284-6289.

22. Box H, Livermore J, Johnson A, et al. Pharmacodynamics of isavuconazole in a dynamic in vitro model of invasive pulmonary Aspergillosis. Antimicrob Agents Chemother. 2015;60:278-287.

23. Seyedmousavi S, Bruggemann RJ, Meis JF, Melchers WJ, Verweij PE, Mouton JW. Pharmacodynamics of isavuconazole in an Aspergillus fumigatus mouse infection model. Antimicrob Agents Chemother. 2015;59:2855-2866.

24. Lepak AJ, Marchillo K, VanHecker J, Diekema D, Andes DR. Isavuconazole pharmacodynamic target determination for Candida species in an in vivo murine disseminated candidiasis model. Antimicrob Agents Chemother. 2013;57:5642-5648.

25. Luo G, Gebremariam T, Lee H, Edwards JE, Jr., Kovanda L, Ibrahim AS. Isavuconazole therapy protects immunosuppressed mice from mucormycosis. Antimicrob Agents Chemother. 2014;58:2450-2453.

26. Majithiya J, Sharp A, Parmar A, Denning DW, Warn PA. Efficacy of isavuconazole, voriconazole and fluconazole in temporarily neutropenic murine models of disseminated Candida tropicalis and Candida krusei. J Antimicrob Chemother. 2009;63:161-166.

27. Najvar LK, Wiederhold NP, Bocanegra R, Olivo M, Kirkpatrick WR, Patterson TF. Isavuconazole is effective for the treatment of experimental cryptococcal meningitis. ICAAC Poster M-427, 2014.

28. Schmitt-Hoffmann AH, Richter WF. Isavuconazole is widely distributed in rat tissue. European Congress of Clinical Microbiology and Infectious Diseases (ECCMID); London, UK; March 31-April 3, 2012: P863.

29. Warn PA, Sharp A, Parmar A, Majithiya J, Denning DW, Hope WW. Pharmacokinetics and pharmacodynamics of a novel triazole, isavuconazole: mathematical modeling, importance of tissue concentrations, and impact of immune status on antifungal effect. Antimicrob Agents Chemother. 2009;53:3453-3461.

30. Falci DR, Pasqualotto AC. Profile of isavuconazole and its potential in the treatment of severe invasive fungal infections. Infect Drug Resist. 2013;6:163-174.

31. FDA Advisory Committee Briefing Document. Isavuconazonium Invasive Aspergillosis and Invasive Mucormycosis. 2015. Available from: http://www.fda.gov/downloads/AdvisoryCommittees/ CommitteesMeetingMaterials/Drugs/Anti-InfectiveDrugsAdvisoryC ommittee/UCM430748.pdf. Accessed January 15, 2016.

32. Astellas Pharma US. Prescribing information for Cresemba 2015; 2015. Available from: www.astellas.us/docs/cresemba.pdf. Accessed January 15, 2016.

33. Schmitt-Hoffmann A, Roos B, Maares J, et al. Multiple-dose pharmacokinetics and safety of the new antifungal triazole BAL4815 after intravenous infusion and oral administration of its prodrug, BAL8557, in healthy volunteers. Antimicrob Agents Chemother. 2006;50: 286-293.

34. Ananda-Rajah MR, Kontoyiannis D. Isavuconazole: a new extended spectrum triazole for invasive mold diseases. Future Microbiol. 2015; 10:693-708.

35. Gubbins PO. Triazole antifungal agents drug-drug interactions involving hepatic cytochrome P450. Expert Opin Drug Metab Toxicol. 2011;7: 1411-1429.

36. Albengres E, Le Louet H, Tillement JP. Systemic antifungal agents. Drug interactions of clinical significance. Drug Saf. 1998;18:83-97.

37. Baciewicz AM, Chrisman CR, Finch CK, SelfTH. Update on rifampin, rifabutin, and rifapentine drug interactions. Curr Med Res Opin. 2013;29:1-12. 
38. Bates DW, Yu DT. Clinical impact of drug-drug interactions with systemic azole antifungals. Drugs Today (Barc). 2003;39:801-813.

39. Bruggemann RJ, Alffenaar JW, Blijlevens NM, et al. Clinical relevance of the pharmacokinetic interactions of azole antifungal drugs with other coadministered agents. Clin Infect Dis. 2009;48:1441-1458.

40. Chan JD. Pharmacokinetic drug interactions of vinca alkaloids: summary of case reports. Pharmacotherapy. 1998;18:1304-1307.

41. Crane JK, Shih HT. Syncope and cardiac arrhythmia due to an interaction between itraconazole and terfenadine. Am J Med. 1993;95: 445-446.

42. Dannaoui E, Schwarz P, Lortholary O. In vitro interactions between antifungals and immunosuppressive drugs against zygomycetes. Antimicrob Agents Chemother. 2009;53:3549-3551.

43. Depont F, Vargas F, Dutronc H, et al. Drug-drug interactions with systemic antifungals in clinical practice. Pharmacoepidemiol Drug Saf. 2007;16:1227-1233.

44. Eiden C, Peyriere H, Tichit R, et al. Inherited long QT syndrome revealed by antifungals drug-drug interaction. $J$ Clin Pharm Ther. 2007;32:321-324.

45. Feldstein AC, Smith DH, Perrin N, et al. Reducing warfarin medication interactions: an interrupted time series evaluation. Arch Intern Med. 2006;166:1009-1015.

46. Gubbins PO. Mould-active azoles: pharmacokinetics, drug interactions in neutropenic patients. Curr Opin Infect Dis. 2007;20 579-586.

47. Kramer MR, Amital A, Fuks L, Shitrit D. Voriconazole and itraconazole in lung transplant recipients receiving tacrolimus (FK 506): efficacy and drug interaction. Clin Transplant. 2011;25:E163-167.

48. Narreddy S, Manavathu E, Chandrasekar PH, Alangaden GJ, Revankar SG. In vitro interaction of posaconazole with calcineurin inhibitors and sirolimus against zygomycetes. JAntimicrob Chemother. 2010;65:701-703.

49. Nivoix Y, Ubeaud-Sequier G, Engel P, Leveque D, Herbrecht R. Drug-drug interactions of triazole antifungal agents in multimorbid patients and implications for patient care. Curr Drug Metab. 2009;10: 395-409.

50. Sathiapalan RK, El-Solh $\mathrm{H}$. Enhanced vincristine neurotoxicity from drug interactions: case report and review of literature. Pediatr Hematol Oncol. 2001;18:543-546.

51. Steinbach WJ, Schell WA, Blankenship JR, Onyewu C, Heitman J, Perfect JR. In vitro interactions between antifungals and immunosuppressants against Aspergillus fumigatus. Antimicrob Agents Chemother. 2004;48:1664-1669.

52. Purkins L, Wood N, Kleinermans D, Nichols D. Voriconazole potentiates warfarin-induced prothrombin time prolongation. Br J Clin Pharmacol. 2003;56(Suppl 1):24-29.

53. Kim T, Jancel T, Kumar P, Freeman AF. Drug-drug interaction between isavuconazole and tacrolimus: a case report indicating the need for tacrolimus drug-level monitoring. J Clin Pharm Ther. Epub 2015 Aug 6.

54. Andes D, Pascual A, Marchetti O. Antifungal therapeutic drug monitoring: established and emerging indications. Antimicrob Agents Chemother. 2009;53:24-34.

55. Walsh TJ, Anaissie EJ, Denning DW, et al. Treatment of aspergillosis: clinical practice guidelines of the Infectious Diseases Society of America. Clin Infect Dis. 2008;46:327-360.

56. Cornely OA, Arikan-Akdagli S, Dannaoui E, et al. ESCMID and ECMM joint clinical guidelines for the diagnosis and management of mucormycosis 2013. Clin Microbiol Infect. 2014;20(Suppl 3): $5-26$.

57. Maertens JA, Raad, II, Marr KA, et al. Isavuconazole versus voriconazole for primary treatment of invasive mould disease caused by Aspergillus and other filamentous fungi (SECURE): a phase 3, randomised-controlled, non-inferiority trial. Lancet. Epub 2015 Dec 9.

58. Marty FM, Ostrosky-Zeichner L, Cornely OA, et al. Isavuconazole treatment for mucormycosis: open-label trial and contemporaneous casecontrol analysis. Lancet Infect Dis. Epub 2016.
59. De Pauw B, Walsh TJ, Donnelly JP, et al. Revised definitions of invasive fungal disease from the European Organization for Research and Treatment of Cancer/Invasive Fungal Infections Cooperative Group and the National Institute of Allergy and Infectious Diseases Mycoses Study Group (EORTC/MSG) Consensus Group. Clin Infect Dis. 2008; 46:1813-1821.

60. Oude Lashof AM, Sobel JD, Ruhnke M, et al. Safety and tolerability of voriconazole in patients with baseline renal insufficiency and candidemia. Antimicrob Agents Chemother. 2012;56:3133-3137.

61. Thompson GR 3rd, Lewis JS 2nd. Pharmacology and clinical use of voriconazole. Expert Opin Drug Metab Toxicol. 2010;6:83-94.

62. McLaughlin JM, Equils O, Somerville KT, et al. Risk-adjusted relationship between voriconazole utilization and non-melanoma skin cancer among lung and heart/lung transplant patients. Transpl Infect Dis. 2013;15:329-343.

63. Zwald FO, Brown M. Skin cancer in solid organ transplant recipients: advances in therapy and management: part I. Epidemiology of skin cancer in solid organ transplant recipients. $J$ Am Acad Dermatol. 2011;65:253-261; quiz 62.

64. Zwald FO, Spratt M, Lemos BD, et al. Duration of voriconazole exposure: an independent risk factor for skin cancer after lung transplantation. Dermatol Surg. 2012;38:1369-1374.

65. Wermers RA, Cooper K, Razonable RR, et al. Fluoride excess and periostitis in transplant patients receiving long-term voriconazole therapy. Clin Infect Dis. 2011;52:604-611.

66. Thompson GR 3rd, Bays D, Cohen SH, Pappagianis D. Fluoride excess in coccidioidomycosis patients receiving long-term antifungal therapy: an assessment of currently available triazoles. Antimicrob Agents Chemother. 2012;56:563-564.

67. Allen KC, Sanchez CJ Jr, Niece KL, Wenke JC, Akers KS. Voriconazole enhances the osteogenic activity of human osteoblasts in vitro through a fluoride-independent mechanism. Antimicrob Agents Chemother. 2015;59:7205-7213.

68. Guinea J, Pelaez T, Recio S, Torres-Narbona M, Bouza E. In vitro antifungal activities of isavuconazole (BAL4815), voriconazole, and fluconazole against 1,007 isolates of zygomycete, Candida, Aspergillus, Fusarium, and Scedosporium species. Antimicrob Agents Chemother. 2008;52:1396-1400

69. Perkhofer S, Lechner V, Lass-Florl C; European Committee on Antimicrobial Susceptibility Testing. In vitro activity of isavuconazole against Aspergillus species and zygomycetes according to the methodology of the European Committee on Antimicrobial Susceptibility Testing. Antimicrob Agents Chemother. 2009;53: $1645-1647$.

70. Ostrosky-Zeichner LIN, Rodriguez J, Chen E, Paetznick V. Comparative in vitro activity of isavuconazole (ISA) against medically important yeasts and moulds. In: Programs and Abstracts of the 49th Annual ICAAC; 2009; San Francisco: Poster M-1707.

71. Curfs-Breuker IM D-OY, Endtz HP, Verweij PE, Meis JF. In vitro activity of caspofungin compared with six other antifungal agents against 239 prospectively collected clinical Aspergillus isolates from the Netherlands. In: Programs and Abstracts of the 3rd Advances Against Aspergillosis Annual Meeting; 2008; Miami: Poster 37.

72. Cuenca-Estrella AGLA, Buitrago MJ, Mellado E, Rodriguez-Tudela JL. BAL4815 a new antifungal agent: Determination of quality control limits for susceptibility testing by methods of the European Committee on Antimicrobial Susceptibility Testing (EUCAST). In: Programs and abstracts of the 46th Annual ICAAC Annual Meeting; 2006; San Francisco: Poster M-1571.

73. Pelaez T GB, Guinea J, Sanchez-Cambronero L, et al. Assessment of the antifungal susceptibility of Aspergillus terreus over an 18-year period in a general hospital. In: Programs and abstracts of the 49th Annual ICAAC Annual Meeting; 2009; San Francisco: Poster M1714.

74. Guinea PT, Gama B, Flores R, et al. Is Aspergillus nidulans susceptible to all antifungal agents? In vitro activity of and updated panel of antifungal agents against 63 clinical isolates. In: Programs and abstracts of the 19th European Congress of Clinical Microbiology and Infectious Diseases (ECCMID); Helsinki; 2009. Oral Presentation. 
75. Verweij PE, Gonzalez GM, Wiedrhold NP, et al. In vitro antifungal activity of isavuconazole against 345 mucorales isolates collected at study centers in eight countries. J Chemother. 2009;21:272-281.
76. Gonzalez GM. In vitro activities of isavuconazole against opportunistic filamentous and dimorphic fungi. Med Mycol. 2009;47:71-76.

\section{Publish your work in this journal}

Infection and Drug Resistance is an international, peer-reviewed openaccess journal that focuses on the optimal treatment of infection (bacterial, fungal and viral) and the development and institution of preventive strategies to minimize the development and spread of resistance. The journal is specifically concerned with the epidemiology of antibiotic resistance and the mechanisms of resistance development and diffusion in both hospitals and the community. The manuscript management system is completely online and includes a very quick and fair peerreview system, which is all easy to use. Visit http://www.dovepress.com/ testimonials.php to read real quotes from published authors.

Submit your manuscript here: http://www.dovepress.com/infection-and-drug-resistance-journal 